\title{
Clinical anophthalmos
}

\author{
M O'KEEFE, M WEBB, R C PASHBY, AND R D WAGMAN \\ From the Department of Ophthalmology, the Hospital for Sick Children, Toronto, Canada
}

SUMmary We report on 15 patients (10 boys, 5 girls) with clinical anophthalmos. Two of them had bilateral anophthalmos, 10 had systemic anomalies, and six had abnormalities of their remaining eye. Only two appeared to have an associated underlying aetiology. Fourteen patients underwent orbital reconstruction or socket enlargement with varying degrees of success. Our own experience suggests that unnecessary lid procedures should be avoided, but we recommend early prosthetic fittings. We feel that a multidisciplinary approach is necessary to attain useful rehabilitation.

Anophthalmos is the absence of the eye as identified without histological examination. The first large clinical series was described by Treacher Collins,' and isolated cases have subsequently been reported. Recently anophthalmos and genetic microphthalmos have been considered together, ${ }^{2}$ since in most cases CT scans show some remnants of either the globe or the surrounding adnexae. Anophthalmos is a rare condition that the average ophthalmologist may never see. In a prospective study of more than 50000 pregnancies in the USA the incidence of anophthalmos or microphthalmos was found to be only 0.22 per 1000 births, ${ }^{3}$ and the prevalence among blind children was thought to be between 0.6 and $1.9 \% .^{+}$

We describe 15 cases of clinical anophthalmos seen over 16 years at the Hospital for Sick Children in Toronto. There is no clear-cut approach to treatment. A variety of surgical techniques have been tried. We outline our own experience in the management of this condition.

\section{Patients and methods}

Fifteen patients (10 of them boys) (Table 1$)$ who presented at birth or shortly thereafter to the Hospital for Sick Children, Toronto, were reviewed. All of them were assessed by specialists in the departments of ophthalmology, neurosurgery, plastic surgery, genetics, and psychology.

Correspondence to Mr Michacl O'Keefe, FRCS, The Children's Hospital, Temple Street, Dublin 1, Eire.

\section{Results}

Two boys had bilateral clinical anophthalmos. One of them was a healthy, full-term, otherwise normal baby who was referred to our unit shortly after birth. A CT scan showed that both globes were absent, but there was evidence of extraocular muscles (Fig. 1). No hereditary or environmental factors could be implicated and the parents were not related. The child appeared mentally normal. The second bilateral case was a boy born with dysmorphic hands and feet and absence of the septum pellucidum. Unfortunately this patient was lost to follow-up. Six patients with unilateral clinical anophthalmos had anomalies in their existing eyes (Table 2). The two patients with iris colobomas had visual acuities of 20/20 and 20/30. The two with moderately severe microphthalmos had recorded visual acuities of 20/100. Another patient had an epibulbar dermoid cyst and an opaque cornea, and one had nystagmus; both of these patients were blind.

Ten patients had associated systemic anomalies as shown in Table 2 . Five of them were also mentally retarded; of these five, all had midline facial clefts, one had hydrocephalus, and one had transsphenoidal

Table 1 Status of 15 patients (10 boys, 5 girls) with anophthalmos

\begin{tabular}{lr}
\hline Problems identified & No. \\
\hline Unilateral anophthalmos & 13 \\
Bilateral anophthalmos & 2 \\
Systemic abnormalitics & 10 \\
Abnormalities of existing cyc & 6 \\
\hline
\end{tabular}






Fig. 1 CT scan showing absent globes with the presence of extraocular muscle.

encephalocele. One patient had a right facial dysplasia and Klippel-Feil syndrome.

Genetic consultation was obtained in 12 cases, but no hereditary basis for the anomalies was found. Maternal rubella was a suspected factor in two patients with unilateral anophthalmos and systemic anomalies. In one other case the parents reported contact with pesticide spray during the first trimester.

The two patients with hydrocephalus and the patient with the transsphenoidal encephalocele had early surgical intervention. All patients with midline facial clefts (Fig. 2) had early and follow-up surgery, and both patients with cardiac anomalies underwent cardiac surgery.

Table 2 Congenital anomalies associated with anophthalmos in 15 patients

\begin{tabular}{lc}
\hline & No. \\
\hline Abnormalities of the existing eye & \\
Iris colobomata & 2 \\
Microphthalmos & 2 \\
Opaque cornea and epibulbar dermoid & 1 \\
Nystagmus & 1 \\
Associated systemic anomalies & \\
Midline facial clefts & 6 \\
Hydrocephalus & 2 \\
Absence of septum pellucidum & 1 \\
Transsphenoidal encephalocele & 1 \\
Klippel-Feil syndrome & 1 \\
Finger and foot abnormalitics & 1 \\
Ventricular septal defect & 2 \\
\hline
\end{tabular}

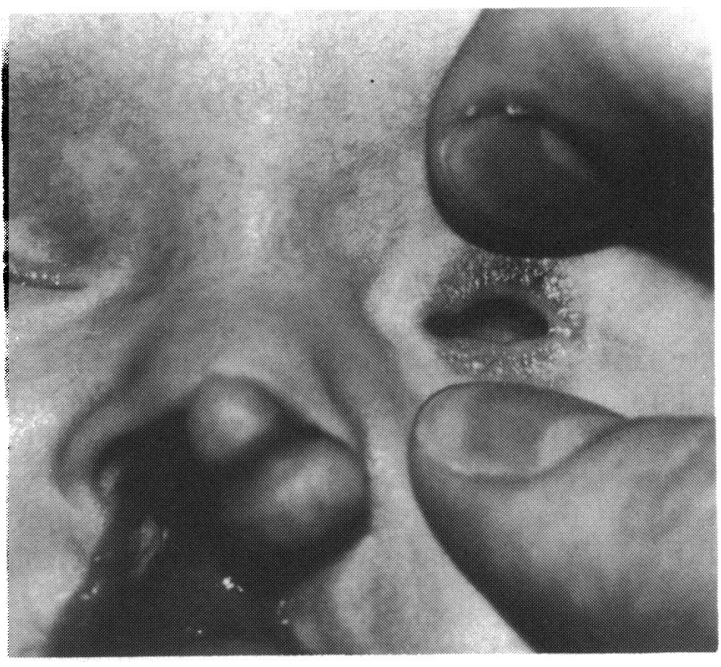

Fig. 2 Patient with unilateral clinical anophthalmos, hydrocephalus, midline facial cleft, and coloboma of the remaining eye.

Orbital reconstruction or socket enlargement was attempted in 14 patients. All received enlarging prosthetic fittings to stimulate growth and development of the bony orbit and adnexae. Six patients had difficulty retaining their prostheses, and four of them underwent multiple surgical procedures, including expansion of palpebral fissures, lid revision, and medial and lateral canthoplasty. Many of the procedures were performed for retention problems but appeared to result in cicatricial tissue formation of the socket up to six months after surgery (Fig. 3). Four patients with unilateral anophthalmos now aged 16 or more show a satisfactory cosmetic appearance.

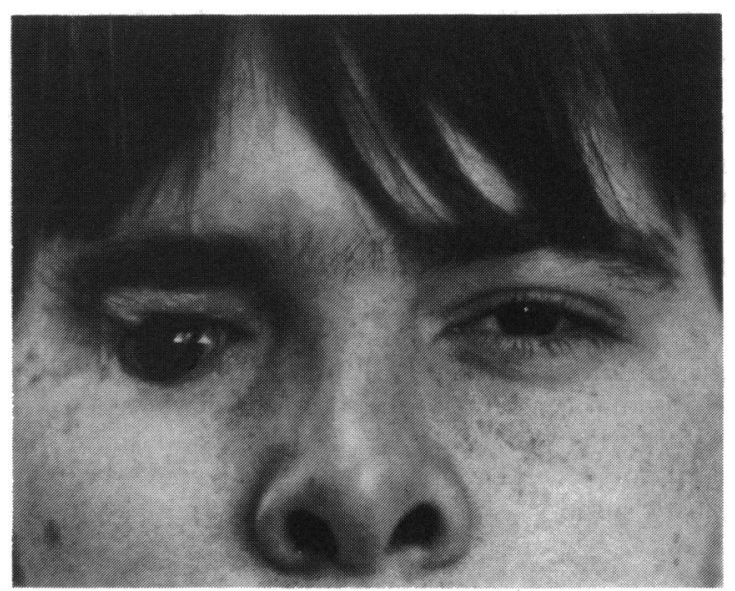

Fig. 3 Patient with multiple lid revisions who continues to have retention problems. 


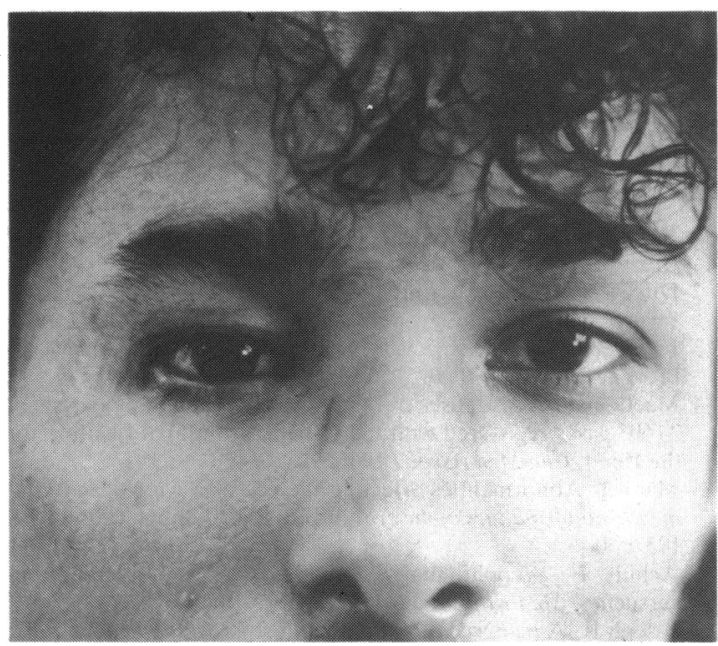

Fig. 4 Patient with early prosthetic therapy without lid revisions and with good cosmetic result.

We believe this results from the fact that no lid revisions were undertaken and prosthetic therapy was initiated within the first year of life (Fig. 4).

Three patients underwent a three dimensional orbital bone expansion combined with prosthetic therapy. The result proved very satisfactory. Two other patients had tissue expanders in the form of synthetic expandible balloons with a reservoir (Fig. 5). This procedure proved unsuccessful in one because the balloons eventually ruptured. The other child with bilateral anophthalmos had the balloons in situ for six months. Periodically additional saline was injected into the reservoir to expand the balloons.



Fig. 5 Orbital balloon expanders.

\section{Discussion}

Anophthalmos is divided into primary, secondary, and consecutive types. ${ }^{5}$ In the primary type the optic primordium does not develop, but there are no generalised gross anomalies of the medullary tube. In secondary anophthalmos there is complete suppression or gross anomalous development of the entire anterior part of the neural tube. In consecutive anophthalmos the optic vesicle has formed but subsequently undergone degeneration. The primary type is thought to be bilateral and sporadic. Otherwise the child is well formed. Secondary anophthalmos results in a severely deformed child and as such does not exist in clinical practice. In the consecutive type a complete absence of the eye has been reported following histological examination in only 10 cases. The globe is also disorganised in cryptophthalmos, where there is complete failure of the eye lids. However its aetiology and its relationship with anophthalmos are obscure. More recently others have used the term microphthalmos to include anophthalmos primarily because some vestige of the globe or surrounding adnexae has developed. The validity of this term has been substantiated by orbital CT scanning. However, we use the term clinical anophthalmos as first coined by Duke-Elder ${ }^{2}$ because it is appropriate to our patients.

Reported associated deformities include craniofacial malformations, polydactyly, cardiac anomalies, and mental retardation. ${ }^{7}$ We found multiple associated anomalies in nine cases. In addition six patients had abnormalities in the existing eye. Two were blind and two had a visual acuity of only 20/100.

The aetiology of clinical anophthalmos has been the subject of speculation. The role of genetics has been fully discussed, ${ }^{\mathrm{x}}$ and patients with autosomal dominant," autosomal recessive, ${ }^{10}$ and sex linked" patterns of inheritance have been reported. However, in most cases the problem is sporadic. In the absence of a history of disease or consanguinity an environmental cause seems probable. Anophthalmos has also been directly related to an attempted mechanical abortion ${ }^{6}$ and to severe maternal vitamin A deficiency. ${ }^{12}$ In our own series two cases occurred in children with rubella syndrome.

Treatment is important to improve the cosmetic appearance, initially for the sake of the parents and later for the child. However, nine of our patients had multiple associated abnormalities. Some required life-saving surgery to treat hydrocephalus and cardiac abnormalities. Additional surgery to correct midline facial clefts further complicated treatment in four cases.

The management of clinical anophthalmos is complex. It is still not certain which overall approach 
provides the best cosmetic result. The obvious orbital discrepancy and unparallel growth of the normal and anophthalmic orbits present a major challenge. Stimulating the growth of the bony orbit via prosthetic therapy at an early age helps to reduce bony orbital discrepancy; however, this technique alone does not provide a satisfactory overall result. Sufficient posterior stability must be achieved to apply anterior pressure to the lids, and this cannot be achieved without surgical intervention. Our experience suggests that unnecessary surgical lid revision should be avoided, but we recommend early prosthetic fittings.

Results achieved with three dimensional orbital expansion have been encouraging. ${ }^{13}$ This procedure can be done when the patient is $2 \frac{1}{2}$ years of age and the orbital bony growth is complete. It is major surgery and requires multidisciplinary co-operation. The other procedure, involving orbital balloon expanders, has been used for some time, but we are not yet in a position to evaluate the benefit. A potential disadvantage is the inability to create posterior orbital expansion.

In patients with associated systemic anomalies more complex treatment is required and more time is needed to achieve an obvious cosmetic improvement. Some of them are blind or mentally retarded or both. These problems may raise questions about the wisdom of an aggressive approach. However, when the children survive, aggressive treatment can improve their quality of life and may be psychologically beneficial for the parents as well. We consider that useful rehabilitation is achieved by a multidisciplinary approach to treatment.
We thank Drs I Munro and J S Crawford for their contribution. This paper was prepared with the assistance of Elizabeth Shapter, the Medical Publications Department, the Hospital for Sick Children, Toronto, and Marese McKiernan, the Mater Private Hospital, Dublin.

\section{References}

1 Treacher Collins E. On anophthalmos R. Lond Ophthalmol Hosp Rep 1887; 11: 429-55.

2 Duke-Elder S, ed. Anophthalmos and extreme microphthalmos. System of ophthalmology. St Louis: Mosby, 1964; 3: 416-23.

3 Vision problems in the US. New York National Socicty to Prevent Blindness, 1980

4 MacDonald AE. Causes of blindness in Canada: an analysis of 24605 cases registered with the Canadian National Institute for the Blind. Can Med Assoc J 1965; 92: 264-79.

5 Mann I. Abnormalities affecting the cye as a whole. Developmental abnormalities of the eye. 2 nd ed. Philadelphia: Lippincott. 1957: 60)-6.

6 Achilis E. Anophthalmos abs Folge eines Intererruptionversuches. Med Klin 1950; 45: 1214-5.

7 Joseph R. A pedigree of anophthalmos. Br J Ophthalmol 1957; 41: $541-3$.

8 Warburg M. Genetics of microphthalmos. Int Ophthalmol 1981; 4: 45-65.

9 Sjörgen T, Larsson T. Microphthalmos and anophthalmos with or without coincident oligophrenia: clinical and genetic statistical study. Acta Psychiatr Neurol 1949; suppl 56: 1-103.

10 Pcarce WG, Nigam S, Rootman J. Primary anophthalmos: histological and genetic features. Can J Ophthalmol 1974: 9: 141-5.

11 Brunquell PJ, Papale JH, Hornton JC. et al. Sex-linked hereditary bilatcral anophthalmos: pathologic and radiologic correlation. Arch Ophthalmol 1984; 102: 108-13.

12 Sarma V. Maternal vitamin A deficiency and fetal microcephaly and anophthalmia: report of a case. Obstet Gynecol 1959; 13: 299-301.

13 Marchac D, Cophignon J. Achard E. Dufourmentel C. Orbital expansion for anophthalmia and microorbitism. Plast Reconstr Surg 1977; 59: 486-91.

Accepted for publication 9 September 1986. 